\title{
Producción de forraje verde hidropónico versus geopónico de cebada (Hordeum vulgare L.)
}

\author{
en ambientes controlados
}

Production of green hydroponic and geoponic forage of barley (Hordeum vulgare L.)

in controlled environments

Blanco-Capia Luis Edgar*, Colque-Pérez Helen, Rosales-Mendoza Marcos Bernardo

\begin{tabular}{l}
\hline DatoS del Articulo \\
\hline Universidad Técnica de Oruro. \\
Facultad de Ciencias Agrarias y Naturales \\
Departamento de Agricultura. \\
Ciudadela Universitaria avenida Dehene \\
Av. Dehene, entre C/ Román Koslowky y \\
C/. León H. Loza. Ciudadela Universitaria \\
Oruro - Estado Plurinacional de Bolivia. \\
Telf: +591 52-61645, +591 25264677. \\
*Dirección de contacto: \\
Luis Edgar Blanco Capia \\
Universidad Técnica de Oruro. \\
Facultad de Ciencias Agrarias y Naturales \\
Departamento de Agricultura. \\
Ciudadela Universitaria avenida Dehene \\
Av. Dehene, entre C/ Román Koslowky y \\
C/. León H. Loza. Ciudadela Universitaria \\
Oruro - Estado Plurinacional de Bolivia. \\
Telf: +591 718 84968. \\
E-mail: luis.blanco.capia@ @gmail.com
\end{tabular}

Palabras clave:

Alimentación ganado, altiplano central boliviano, escasez de forraje,

producción de forraje verde, rendimiento de forraje.

J Selva Andina Biosph. 2019; 7(2):109-117.

\section{Historial del artículo.}

Recibido junio, 2019. Devuelto agosto 2019

Aceptado septiembre, 2019.

Disponible en línea, noviembre 2019.

\begin{tabular}{c} 
Editado por: \\
Selva Andina \\
Research Society \\
\hline
\end{tabular}

\section{Key words:}

Feeding livestock, central bolivian highlands, shortage of fodder, production of green fodder, forage yield.
() 2019. Journal of the Selva Andina Biosphere. Bolivia. Todos los derechos reservados.

\section{Abstract}

The central region of the Bolivian highlands is cattle-raising, in adverse climatic conditions, due to the shortage of food mainly in the winter period, which affects the economy of the producer. The study was carried out in the winter of the Center for Research and Production of Small Animals (CIPAM) of the Faculty of Agricultural and Natural Sciences of the UTO, with the purpose of evaluating the height and performance of the hydroponic green geoponic feeding versus barley. (Hordeum vulgare L.) of the IBTA-80 variety under controlled environmental conditions. At room temperature it fluctuated from 20 to $26^{\circ} \mathrm{C}$, the relative humidity between 15.9 and $24.4 \%$. This produces a viability of $85.8 \pm 2.2 \%$ and is used with a density of $10 \mathrm{~kg} / \mathrm{m}^{2}$. The experimental design was the Random Blocks with hydroponic and geoponic treatments. Hydroponics production is carried out with water without any nutritional solution, in the case of geoponics if a substrate one centimeter high can be used. In the harvest (10 days) the height of the plant is estimated for hydroponic crops with $23.8 \pm$ $0.31 \mathrm{~cm}$ and in geoponic $20.5 \pm 0.30 \mathrm{~cm}$; performance also improved in hydroponics $\left(25.1 \pm 0.25 \mathrm{~kg} / \mathrm{m}^{2}\right)$ compared to geoponics $\left(24.3 \pm 0.35 \mathrm{~kg} / \mathrm{m}^{2}\right)$, but there is also a correlation $(\mathrm{r}=0.6196)$ between height and performance $(\mathrm{p}<0.01)$. The technique of producing hydroponic green fodder changes under adverse climatic conditions as a technical and economical alternative to feed livestock. 


\section{Introducción}

Bolivia es una región con variaciones climáticas y edafológicas, esto hace que la agricultura sea limitada cuando se habla de seguridad alimentaria (SA), debido a diferentes prácticas, como sistemas de cultivo rudimentarios ${ }^{1}$, escasez de agua, exceso de sales en el agua y suelo ${ }^{2}$ particularmente en la zona altiplánica. La actividad económica principal del Altiplano central de Bolivia es la ganadería, entre ovinos y llamas. ${ }^{3}$ Sin embargo, la disponibilidad de forraje en el sistema tradicional de alimentación animal basado en el pastoreo extensivo a campo abierto, enfrenta una serie de contrastes asociados al cambio climático y a la crisis mundial del agua. ${ }^{4}$

Las familias campesinas del altiplano boliviano se dedican a la actividad agrícola y ganadera de pequeña escala, de condiciones climáticas adversas que reducen su capacidad productiva, ${ }^{5,6}$, mucho más en época de invierno, los pobladores deben implementar acciones preventivas para la provisión de agua de uso agrícola ${ }^{7}$.

Condiciones donde el ganado no tiene suficiente alimento, disminuyendo los índices productivos y reproductivos, aspectos que incide directamente en la economía del productor rural y urbano. Consiguientemente, es imprescindible adoptar nuevos métodos de producción de forraje para ganado.

El forraje verde hidropónico $(\mathrm{FVH})$, es la producción de biomasa forrajera en periodos cortos a la $\operatorname{cosecha}^{8,9}$ y el forraje verde geopónico (FVG) son alternativas de cultivo para la alimentación del ganado, porque constituyen un forraje verde de calidad, libre de malezas, plagas y enfermedades ${ }^{10-13}$, además inocuos y de alta palatabilidad. ${ }^{1,2,10}$ El forraje hidropónico es apto para la alimentación de ganado, sobre todo durante tiempos de escasez de forraje verde $^{10,14}$ que permitirá el rendimiento por el peso consumido y la asimilación del alimento sean altos.
Para la producción de este tipo de agricultura se requiere ambientes controlados, pudiéndose cultivar en espacios reducidos e incluso en condiciones climáticas adversas ${ }^{15}$, además, estas técnicas permiten planificar la cantidad de producción deseada y en cualquier época del año.

Este método productivo ofrece amplias ventajas ya que se puede controlar de mejor manera el desarrollo de un cultivo principalmente en lo referente a plagas, enfermedades y factores climáticos en tiempos y épocas difíciles como el invierno ${ }^{13}$ y la falta de terrenos, de estas técnicas de producción pueden beneficiarse varios sectores productivos pecuarios. ${ }^{13,16}$ En varios países ya se ha estudiado muchas especies para la producción de forraje verde hidropónico y han dado resultados óptimos en cuanto a sus propiedades nutricionales, por esta razón es importante aplicar estas prácticas en nuestra región. ${ }^{9,2}$

Existen diferentes modalidades de producción de FVH, la técnica básica consiste en germinar semillas de cereales a altas densidades de siembra, con el propósito de obtener un forraje de 20 a $30 \mathrm{~cm}$ en un periodo de 8 a 12 días. ${ }^{17}$ El FVH se produce en ausencia del suelo suelo ${ }^{9,13,18}$, el mismo es reemplazado por agua con los nutrientes minerales esenciales disueltos en ella, a la cual se le denomina solución nutritiva $^{1,11,19,20,21}$ y en condiciones protegidas donde se controlan algunas variables ambientales como la luz, temperatura y humedad. ${ }^{12}$

Por las consideraciones mencionadas la presente investigación realizada en la región central del Altiplano boliviano, busca evaluar la altura de planta al momento de la cosecha y el rendimiento en forraje verde de cebada (Hordeum vulgare L.) a través de técnicas hidropónica y geopónica en ambientes controlados, para la alimentación de ganado menor. 


\section{Materiales y métodos}

El experimento fue conducido en el primer semestre del 2017, en un invernadero del Centro de Investigación y Producción de Animales Menores (CIPAM) de la Facultad de Ciencias Agrarias y Naturales - Universidad Técnica de Oruro, ubicada en la ciudad de Oruro, Bolivia a una altura de 3712 msnm. En este ambiente controlado se registró una temperatura ambiente promedio entre 20 a $26{ }^{\circ} \mathrm{C}$ y la humedad relativa de 15.9 a $24.4 \%$, condiciones de ambiente consideradas moderadas y aptas para que el FVH/FVG puedan desarrollarse normalmente. $^{22}$

Se utilizó semilla de cebada (Hordeum vulgare L.) forrajera variedad IBTA-80 procedente del municipio de Caracollo con un porcentaje de germinación $85.8 \pm 2.2 \%$, evaluándose el tamaño, homogeneidad en la forma y color, sin defectos y libre de microorganismos. ${ }^{11}$ Se utilizaron ocho bandejas de $720 \mathrm{~cm}^{2}$ $(24 \times 30 \mathrm{~cm})$ por tratamiento, con una densidad de $10 \mathrm{~kg}$ de semilla $/ \mathrm{m}^{2}$, resultando $720 \mathrm{~g} / \mathrm{bandeja}$, figura 1 .

La semilla se desinfectó con hipoclorito de sodio al $1 \%$ por un periodo de $30 \mathrm{~min}$, y luego fueron lavadas con agua fría repetidamente. ${ }^{10}$ Posteriormente se hidrataron en agua por un periodo de $24 \mathrm{~h}$ a temperatura ambiente. La semilla hidratada pasó a su pre germinación, en bandejas forradas con plástico negro por dos días aproximadamente, posteriormente se espera hasta su máximo crecimiento, que fue a los 10 días. ${ }^{9}$ El riego se efectuó dos veces por aspersión en forma diaria.

Se realizaron seis pruebas, evaluándose al momento de la cosecha la altura de 20 plantas (elegidas al azar) y el rendimiento de forraje verde parte aérea por bandeja estimado por $\mathrm{m}^{2}$.

Se empleó el diseño estadístico bloques al azar, con dos cultivos (hidro y geopónico), con ocho repeti- ciones y seis bloques (pruebas de producción), según modelo siguiente. Las diferencias entre cultivos fueron determinadas mediante el análisis de varianza con un nivel de significancia del 0.01 y con el supuesto de distribución Gamma se empleó el procedimiento GENMOD del paquete estadístico SAS v. 9.2. ${ }^{23,24}$ Por otro lado, se efectuó el análisis de correlación simple entre dichas variables.

Dónde:

$$
\eta_{i j k}=\log i t\left(\frac{\pi_{i j k}}{1-\pi_{i j k}}\right)=\eta+\beta_{j}+\tau_{i}
$$

$\mathrm{i}=1,2, \ldots \mathrm{a}$ de factor cultivo (hidropónico y geopónico)

$\mathrm{j}=1,2, \ldots \mathrm{b}$ de bloques

$\mathrm{k}=1,2, \ldots \mathrm{r}$ de número de réplicas

$\eta_{i j k}=$ Valor generalizado sobre la relación de la ocurrencia de plantas $\pi_{i j k}$ sobre la no ocurrencia de la unidad experimental ubicada en el j-ésimo bloque y que recibe el

$\eta=$ Constante i-ésimo cultivo y k-ésimo número de repeticiones

$\beta_{\mathrm{j}}=$ Efecto fijo j-ésimo bloque

$\tau_{i}=$ Efecto fijo del i-ésimo cultivo (hidropónico y geopónico)

Figura 1 Distribución de bandejas de germinación de forraje verde
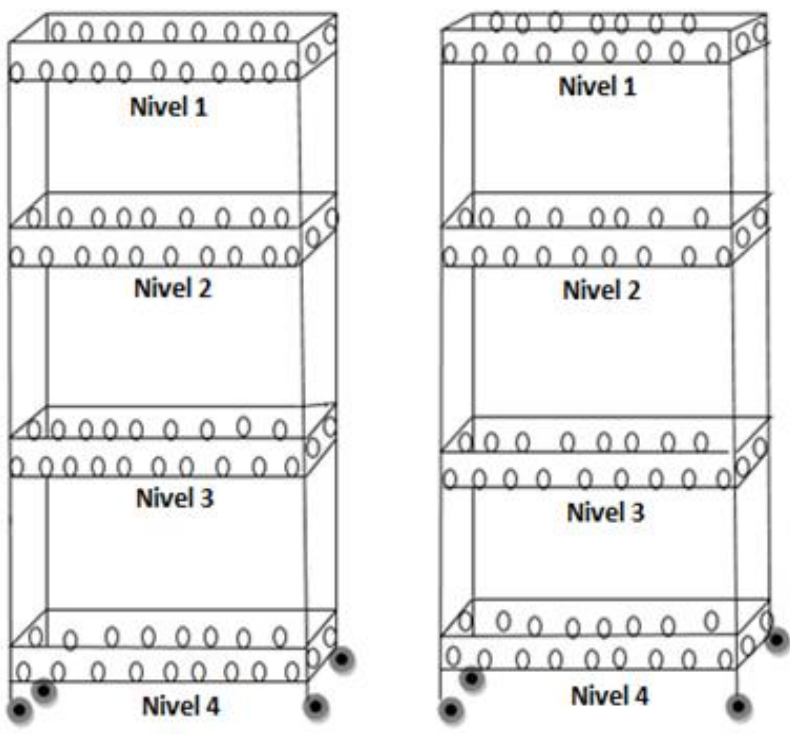

\section{Resultados}

Altura de planta a la cosecha. Se leyeron 96 observaciones distribuidas en dos tratamientos (hidropónico y geopónico) y seis bloques. Según los estadísticos de Wald para análisis de tipo 3, se determina 
para el factor bloque (valor mayor a 0.05) existe variación entre los distintos bloques. La prueba de efectos fijos, determina que el valor de $\mathrm{F}$ es igual a 256.05 a una probabilidad <.0001, mucho menor al nivel del 0.01, consiguientemente existe diferencia significativa a la probabilidad del 0.01 , entonces con $99 \%$ de confiabilidad se infiere que las alturas entre los cultivos hidropónicos y geopónicos son diferentes (figura 2). La prueba de diferencia de medias de mínimos cuadrados demuestra que ambos tratamientos mantienen diferencia, logrando a los 10 días de la siembra alturas de $23.8 \pm 0.31 \mathrm{~cm}$ para hidropónico y $20.5 \pm 0.30 \mathrm{~cm}$ en geopónico (figura 2).

Figura 2 Diferencia de efectos principales (hidroponía y geoponía) para altura de planta y rendimiento de forraje verde de cebada (Hordeum vulgare $\mathrm{L}$.), evaluadas al momento de la cosecha
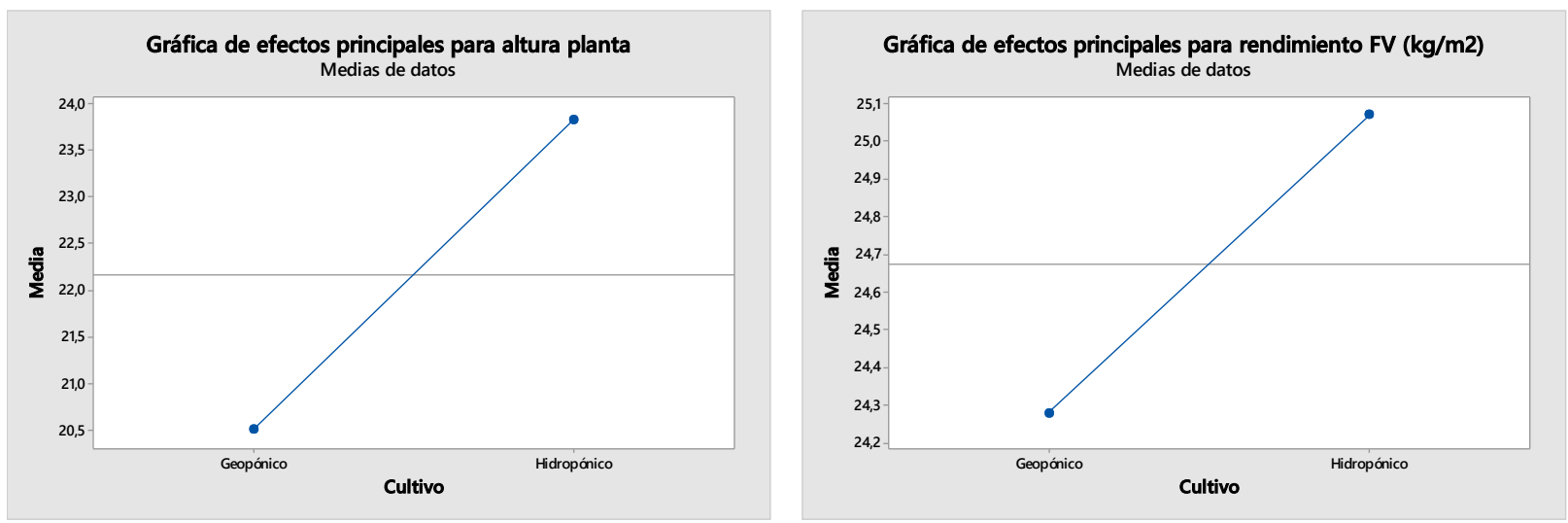

Figura 3 Altura de planta y rendimiento de forraje verde de cebada (Hordeum vulgare L.) producidas en hidroponía y geoponía, evaluadas al momento de la cosecha

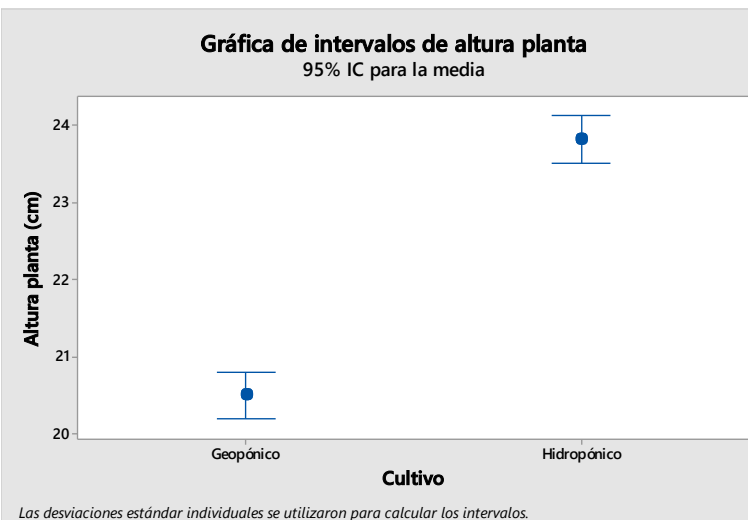

Las desviaciones estándar individuales se utilizaron para calcular los intervalos

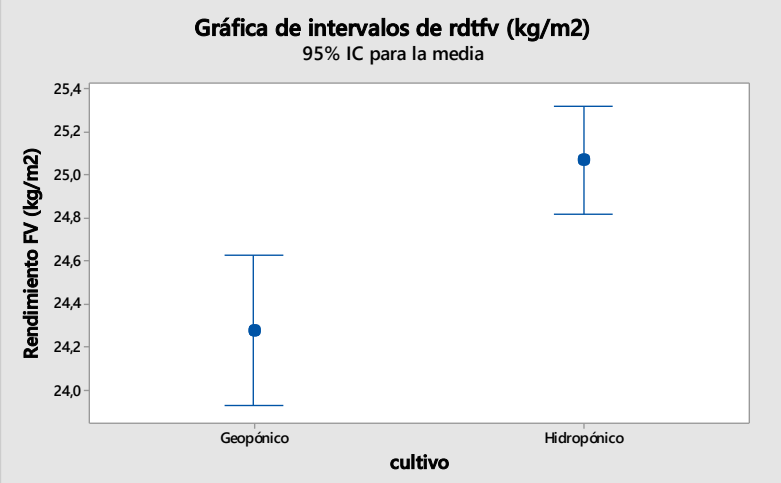

Las desviaciones estándar individuales se utilizaron para calcular los intervalos
Rendimiento de forraje verde por superficie. Se leyeron 96 observaciones distribuidas en dos tratamientos (hidropónico y geopónico) y seis bloques. Según los estadísticos de Wald para análisis de tipo 3 , se determina para el factor bloque (valor mayor a
0.05) existe variación entre los distintos bloques. La prueba de efectos fijos, determina que el valor de $\mathrm{F}$ es igual a 15.47 a una probabilidad <.0001, mucho menor al nivel del 0.01 , consiguientemente existe diferencia significativa a la probabilidad del 0.01 , 
entonces con $99 \%$ de confiabilidad se infiere que los rendimientos de forraje verde por superficie entre los cultivos hidropónicos y geopónicos son diferentes. La diferencia de medias de mínimos cuadrados (figura 2) se evidencia que mediante la técnica hidropónica se obtuvo un rendimiento de $25.1 \pm 0.25$ $\mathrm{kg} / \mathrm{m}^{2}$, en comparación a $24.3 \pm 0.35 \mathrm{~kg} / \mathrm{m}^{2}$ para el cultivo geopónico (figura 3 ).

Figura 4 Diagrama de dispersión de altura de planta (cm) versus rendimiento de forraje en verde $\left(\mathrm{kg} / \mathrm{m}^{2}\right)$

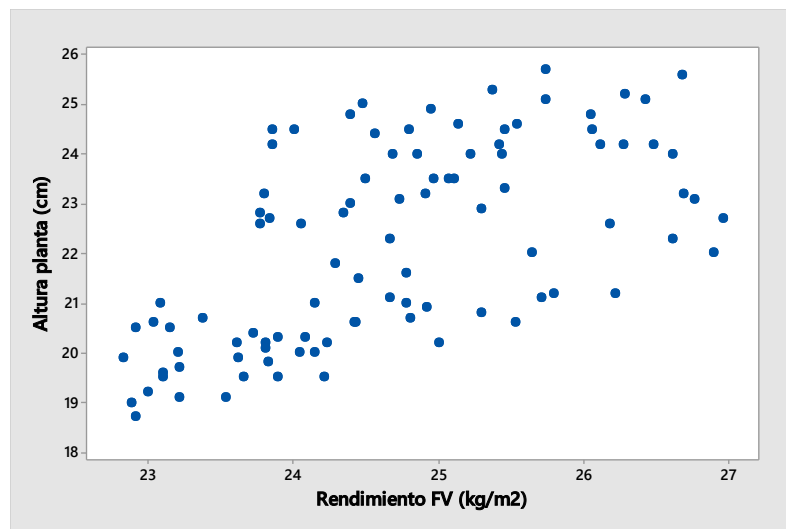

Grado de relación entre altura planta y rendimiento de forraje verde. Según la figura 4 , respecto a la dispersión entre las variables altura de planta y rendimiento de forraje verde por superficie existe relación, esto corroborado por el análisis de correlación asumiendo que existe correlación $(r=0.6196)$ entre dichas variables al nivel de probabilidad del 0.01; en otras palabras, la altura de la planta influye en $61.96 \%$ al rendimiento de forraje verde por superficie.

\section{Discusión}

El análisis estadístico de altura de planta del cultivo de cebada a los 10 días de siembra, reporta magnitudes de $23.8 \pm 0.31 \mathrm{~cm}$ para el forraje verde hidropónico y $20.5 \pm 0.30 \mathrm{~cm}$ para el forraje verde geopónico. Por otro lado, también se determinó diferencia de medias en el rendimiento de forraje verde mediante las técnicas hidropónica y geopónica, obteniéndose valores de $25.1 \pm 0.25 \mathrm{~kg} / \mathrm{m}^{2}$ y $24.3 \pm 0.35$ $\mathrm{kg} / \mathrm{m}^{2}$ respectivamente, a una densidad de $10 \mathrm{~kg}$ de semilla/ $\mathrm{m}^{2}$.

En otro estudio, el rendimiento promedio de cebada fue de $32.8 \mathrm{~kg} / \mathrm{m}^{2}$ y una altura de $21.7 \mathrm{~cm}$ a una densidad de $3.5 \mathrm{~kg} / \mathrm{m}^{2}$, en un periodo de ocho días, ${ }^{8}$ estudio que se efectuó en invernaderos en la Universidad Autónoma de Chapingo-México. Por otro lado, al incorporar diferentes niveles de azufre (hasta $4 \mathrm{ppm} / \mathrm{m}^{2}$ ) como aditivo en la producción del forraje verde hidropónico de cebada, se determinó una biomasa de $34.9 \pm 0.5 \mathrm{~kg} / \mathrm{m}^{2}$ y altura de tallo de $14.61 \pm 1.38 \mathrm{~cm}$, se evaluó también la digestibilidad aparente de fibra cruda y extracto etéreo en cuyes, determinándose que fueron significativamente influenciados por los niveles de azufre. ${ }^{10}$

Por otro lado, se estudió el efecto de diferentes asociaciones entre arveja con cebada y trigo concluyéndose que el porcentaje de materia seca y rendimiento de forraje verde fue estadísticamente similar en las asociaciones arveja-cebada y arveja-trigo; los porcentajes de materia orgánica y proteína cruda (22.37\%) fueron afectados por la asociación y por el nivel de proporción leguminosa/gramínea; la proporción de leguminosa/gramínea no afectó la altura de planta del cultivo hidropónico. ${ }^{2}$

Asimismo, se mencionan algunas investigaciones en hidroponía en otras especies forrajeras como el maíz. Al utilizar fertilizante inorgánico en variedades de maíz en hidroponía se determinó mayor peso (1.60 kg en bandejas de 40 x $50 \mathrm{~cm}$ ) del maíz blanco con $12.49 \mathrm{~cm}$ de longitud. ${ }^{22}$ En cambio, con la utilización de abono orgánico como el biol presentó una mayor altura de planta $(25.74 \mathrm{~cm})$ y 26.12 $\mathrm{kg} / \mathrm{m}^{2}{ }^{15}$ Utilizando el riego cada 24 horas los valores fueron mayores en altura media de $30.45 \pm 4.5$ $\mathrm{cm}$, un rendimiento $2.5335 \pm 0.3 \mathrm{Kg}$ y un $80.5 \%$ de 
germinación. ${ }^{11}$ En variedades de maíz Morocho Blanco y Amarillo, sometidas a diferentes niveles de silicio $(0,0.025$ y $0.5 \mathrm{~mL} / \mathrm{L})$ también en condiciones de invernadero, se obtuvo que las alturas de plantas y el rendimiento de forraje fresco al nivel de Silicio $0,025 \mathrm{~mL} / \mathrm{L}$ fueron los mayores promedios. ${ }^{16}$ Con base en la nutrición de nitrógeno en forma de nitrato incrementó la altura, el rendimiento por metro cuadrado, el contenido proteico a $17.9 \%$ y una concentración de nitrato de $3348.2 \mathrm{mg} / \mathrm{kg}$, la cual se considera segura para la alimentación animal. ${ }^{21}$ Asimismo, los días a la cosecha afectan el rendimiento, el mejor es a los 16 días, la calidad nutrimental del forraje en cuanto al contenido de proteína cruda y fibra ácido detergente son mejores. ${ }^{25}$

Los aumentos en la frecuencia de circulación de solución nutritiva, priorizó el desarrollo de la biomasa del brote en detrimento de la raíz. ${ }^{26}$ La fertilización con solución nutritiva orgánica incrementó el rendimiento ( $\geq 19 \%$ ), asimismo el uso eficiente del agua ( $250 \%$ ) y la calidad nutrimental. ${ }^{20}$ Por lo que se confirma el potencial de uso sustentable del lixiviado de vermicomposta en la producción de FVH en zonas áridas, que al ser un producto derivado de la ganadería (estiércoles), reducen los costos y la contaminación agroambiental. ${ }^{20,27}$ Por otro lado, los parámetros productivos del forraje verde, son mejores cuando se cultiva a un $75 \%$ de solución nutritiva con una edad de cosecha de 12 días, determinándose una conversión a biomasa de $5.27 \pm 0.06 \mathrm{~kg}{ }^{28} \mathrm{En}$ condiciones de iluminación natural deficiente, el método de cultivo con papel absorbente fue más eficiente para el rendimiento y la cantidad de proteína bruta. ${ }^{29}$ Entonces se obtienen grandes rendimientos de biomasa, producidos en pequeñas áreas sin requerir gran cantidad de maquinaria y un uso más eficiente del agua para riego. ${ }^{18}$ Los nutrientes minerales esenciales se suministran a las plantas a través de una solución nutritiva equilibrada para satisfacer sus necesidades nutricionales. ${ }^{30,31}$
En la avena se evaluaron a través de tres ensayos en condiciones de desierto en el sector Pampa del Tamarugal-Chile, determinándose el mejor tratamiento el remojo por $12 \mathrm{~h}$ a una densidad de $6.4 \mathrm{~kg} / \mathrm{m}^{2}$, el mejor tiempo de cosecha fue a los 10 días, logrando $36.86 \%$ materia seca, $14.79 \%$ proteína bruta, $18.77 \%$ fibra cruda y una mejor condición sanitaria de forraje. ${ }^{32}$

Se incursionó la hidroponía en el cultivo de la papa, determinando incremento en la tasa de crecimiento del cultivo, así como la biomasa y número de tubérculos mayores de $10 \mathrm{~mm}$, por unidad de superficie en invernadero cuando la densidad de población es moderada ( 8 plantas $\left./ \mathrm{m}^{2}\right) .{ }^{33}$ Respecto al sustrato compuesto de fibra de cáscara de coco es más adecuado para el cultivo de lechuga hidropónica, ya que condujo a mayores rendimientos $\left(2.58 \mathrm{~kg} / \mathrm{m}^{2}\right){ }^{13}$ Se demostró el potencial de utilización del forraje como fuente de alimentación en la producción animal. ${ }^{4,1}$ Por ejemplo, el forraje de avena hidropónica en la alimentación de conejos mejoró la ganancia de peso diario (a $35.09 \mathrm{~g}$ ), el índice de conversión alimenticia (5.5), además del peso del animal al mercado (3136.5 g) y una buena rentabilidad. ${ }^{9}$ Por otro lado, en la alimentación de borregos con propósitos de engorda, el uso del forraje verde hidropónico se muestra como una alternativa técnica y económicamente viable en relación a otros tipos de dieta. ${ }^{8}$ Respecto a la producción y calidad de leche de cabras fueron mayores al suministrar la dieta compuesta con alfalfa henificada y forraje verde hidropónico de maíz al 30\%. ${ }^{14}$ Consiguientemente la hidroponía para la producción de forraje es adecuada, este insumo es aceptada en la alimentación del ganado lechero. ${ }^{18}$ Además son una buena fuente de minerales para el ganado, como el arroz, a diferencia del maíz que es un material más suculento. ${ }^{34}$ Es destacable mencionar que el uso de forraje verde hidropónico de maíz deshidratado con balanceado 
tuvo excelentes resultados en el rendimiento a la canal de conejos nueva Zelanda. ${ }^{31}$

Siendo la agricultura una actividad de renovación tecnológica, es vital desarrollar nuevas técnicas que permitan un crecimiento y mejoramiento de alternativas productivas disponibles. Por tanto, entre las ventajas de la hidroponía, están la mayor productividad del cultivo, la precocidad, la mejor calidad del producto y su rentabilidad ${ }^{30}$ brindando desarrollo productivo, además viene a ayudar a promover la protección ambiental, al igual que la sustentabilidad. ${ }^{19}$ Especialmente es útil durante períodos de sequía cuando el forraje verde es escaso, por lo que se considera una alternativa para complementar la alimentación del ganado y contrarrestar los efectos de cambio climático en los sectores agrícola y ganadero. ${ }^{12}$ Entonces, en las condiciones del Altiplano central de Bolivia se muestra como una alternativa técnica y económicamente viable para la producción de forraje para la alimentación de ganado en relación a otros tipos de dieta, siendo imprescindible adoptar nuevos métodos de producción de forraje para animales menores y aves de corral. ${ }^{18}$

\section{Conflictos de intereses}

Esta investigación se realizó en el Centro de Investigación y Producción de Animales Menores de la Facultad de Ciencias Agrarias y Naturales - Universidad Técnica de Oruro y no existe ningún tipo de conflicto de intereses.

\section{Agradecimientos}

Los autores agradecen a Facultad de Ciencias Agrarias y Naturales - Universidad Técnica de Oruro, por el apoyo técnico, científico y logístico realizado a la presente investigación.

\section{Aspectos Éticos}

La investigación ha sido aprobada por la Dirección de Postgrado e Investigación Científica de la Facultad de Ciencias Agrarias y Naturales-Universidad Técnica de Oruro y siguió las pautas establecidas para este proceso.

\section{Literatura Citada}

1. Ramírez Víquez C, Soto Bravo F. Efecto de la nutrición mineral sobre la producción de forraje verde hidropónico de maíz. Agron Costarricense 2017;41(2):79-91.

2. Contreras JL, Tunque M, Cordero AG. Rendimiento hidropónico de la arveja con cebada y trigo en la producción de germinados. Rev Investig Vet Perú 2015;26(1):9-19. DOI: http://doi.org/ 10.15381/rivep.v26i1.10910

3. Ministerio de Desarrollo Productivo y Economía Plural del Estado Plurinacional de Bolivia [Internet]. Sistema de Información Territorial de Apoyo a la Producción: Atlas de potencialidades productivas de Bolivia 2019 [citado 22-de octubre de 2018]. Recuperado a partir de: https://siip.pro duccion.gob.bo/repSIIP2/atlas.php

4. Soto Bravo F, Ramírez Víquez C. Efecto de la nutrición mineral en el rendimiento y las características bromatológicas del forraje verde hidropónico de maíz. Pastos y Forrajes 2018;41(2):10613.

5. Estrada Zuñiga AC, Cárdenas Rodríguez J, Naupari Vásquez J, Zapana Pari JG. Capacidad de carga de pastos de puna húmeda en un contexto de cambio climático. Rev Investig Altoandin 2018;20(3):361-8. DOI: http://doi.org/10.18271/ ria.2018.399

6. Mamani Paredes J, Cotacallapa Gutiérrez FH. Rendimiento y calidad nutricional de avena forra- 
jera en la región de Puno. Rev Investig Altoandin 2018;20(4):385-400. DOI: http://doi.org/10.18 271/ria.2018.415

7. León Ochoa RF, Portuguez Maurtua DM, Chávarri Velarde EA. Modelación de la disponibilidad hídrica del rio Piura-Perú, considerando la incidencia del cambio climático. Rev Investig Altoandin 2019;21(3):182-93. DOI: http://doi.org/ 10.18271/ria.2019.476

8. Sánchez Del Castillo F, Moreno Pérez EC, Contreras Magaña E, Morales Gómez J. Producción de forraje hidropónico de trigo y cebada y su efecto en la ganancia de peso en borregos. Rev Chapingo Ser Hortic 2013;19(4):35-43. DOI: http://doi.org/10.5154/r.rchsh.2012.02.020

9. Fuentes Carmona FF, Poblete Pérez CE, Huerta Pizarro MA. Respuesta productiva de conejos alimentados con forraje verde hidropónico de avena, como reemplazo parcial de concentrado comercial. Acta Agron 2011;60(2):183-9.

10. Quispe Cusi A, Paquiyauri Z, Ramos YV, Contreras JL, Véliz MA. Influencia de niveles de azufre en la producción, composición química bromatológica y digestibilidad del forraje verde hidropónico de cebada (Hordeum vulgare L.). Rev Investig Vet Perú 2016;27(1):31-8. DOI http://doi.org/10.15381/rivep.v27i1.11457

11.Zagal Tranquilino M, Martínez González S, Salgado Moreno S, Escalera Valente F, Peña Parra B, Carrillo Díaz F. Producción de forraje verde hidropónico de maíz con riego de agua cada 24 horas. Abanico Vet 2016;6(1):29-34.

12.Juárez López P, Morales Rodríguez HJ, Sandoval Villa M, Gómez Danés AA, Cruz Crespo E, Juárez Rosete CR, et al. Producción de forraje verde hidropónico. Rev Fuente 2013;4(13);16-26.

13.Jordan RA, Ribeiro EF, De Oliveira FC, Geisenhoff LO, Martins EAS. Yield of lettuce grown in hydroponic and aquaponic systems using different substrates. Rev Bras Eng Agríc Ambient
2018;22(8):525-9. DOI: http://doi.org/10.1590/ 1807-1929/agriambi.v22n8p525-529

14.García Carrillo M, Salas Pérez L, Esparza Rivera JR, Preciado Rangel P, Romero Paredes J. Producción y calidad fisicoquímica de leche de cabras suplementadas con forraje verde hidropónico de maíz. Agron Mesoam 2013;24(1):169-176. DOI: http://www.doi.org/10.15517/am.v24i1.979 $\underline{4}$

15.Ticona Aliaga J, Tito Mamani A. Evaluación de la producción de forraje verde hidropónico de maíz (Zea mays L.), con cuatro tipos de abonos orgánicos bajo ambiente atemperado en la provincia Murillo del Departamento de La Paz. Apthapi 2017;3(2):538-44.

16.González E, Ceballos J, Benavidez O. Producción de forraje verde hidropónico de maíz Zea mays. L. en invernadero con diferentes niveles de silicio. Rev Cienc Agr 2015;32(1):75-83. DOI: DOI: https://doi.org/10.22267/rcia.153201.26

17.Resh HM. Cultivos hidropónicos. Madrid: Ediciones Mundi Prensa; 2001. 558 p.

18. Romero Valdez ME, Córdova Duarte G, Hernández Gallardo EO. Producción de forraje verde hidropónico y su aceptación en ganado lechero. Acta Univ 2009;19(2):11-9. DOI: http://www. doi.org/10.15174/au.2009.93

19.López Elías J. La producción hidropónica de cultivos. Idesia (Arica) 2018;36(2):139-41. DOI: https://doi.org/10.4067/S0718-342920180050008 $\underline{01}$

20.Preciado Rangel P, García Hernández JL, Segura Castruita MA, Salas Pérez L, Ayala Garay AV, Esparza Rivera JR, et al. Efecto del lixiviado de vermicomposta en la producción hidropónica de maíz forrajero. Terra Latinoam 2014;32(4):3338.

21.Maldonado Torres R, Álvarez Sánchez ME, Cristóbal Acevedo D, Ríos Sánchez E. Nutrición mineral de forraje verde hidropónico. Rev Chapingo 
Ser Hortic 2013;19(2):211-23. DOI: http://doi. org/10.5154/r.rchsh.2011.10.053

22.González E, Ceballos J, Benavides O. Producción de forraje verde hidropónico de maiz Zea mays $\mathrm{L}$. en invernadero con diferentes niveles de silicio. Rev Cienc Agr 2015;32(1):75-83. DOI: https:// doi.org/10.22 267/rcia.153201.26

23. Montgomery DC. Diseño y análisis de experimentos [Internet]. México: Limusa Wiley SA de CV; 2004 [citado 22-de octubre de 2018]. 686 p. Recuperado a partir de: https://www.academia. edu/9101936/Dise\%C3\%B1o_y_an\%C3\%A1lisis _de_experimentos_Douglas_C._Montgomery

24. Sas/Stat ${ }^{\circledR}$ 9.2. User's Guide introduction to statistical modeling with SAS/STAT software (Book Excerpt): Cary, North Carolina; 2008. 60 p.

25. Salas Pérez L, Preciado Rangel P, Esparza Rivera JR, Álvarez Reyna VP, Palomo Gil A, Rodríguez Dimas N, et al. Rendimiento y calidad de forraje hidropónico producido bajo fertilización orgánica. Terra Latinoam 2010;28(4):355-60.

26.Campos Junior JE, Santos Junior JA, Bezerra Martins J, de França e Silva EF, Cabral de Almeida CDG. Rocket production in a low cost hydroponic system using brackish water. Rev Caatinga 2018;31(4):1008-16. DOI: http://doi.org/10. 1590/1983-21252018v31n424rc

27.López Aguilar R, Murillo Amador B, Rodríguez Quezada G. El forraje verde hidropónico $(\mathrm{FVH})$ : una alternativa de producción de alimento para el ganado en zonas áridas. Interciencia 2009;34 (2):121-6.
28. Morales Rodríguez HJ, Gómez Danés AA, Juárez López P, Loya Olguín L, Ley de Coss A. Forraje verde hidropónico de maíz amarillo (Zea mays L.) con diferente concentración de solución nutritiva. Abanico Vete 2012;2(3):20-8.

29. Rivera A, Moronta M, Gonzáles Estopiñán M, González D, Perdomo D, García DE, et al. Producción de forraje verde hidropónico de maíz (Zea mays L.) en condiciones de iluminación deficiente. Zootecnia Trop 2010;28(1):33-41.

30.Neto Bezerra E, Barreto Paes L. As técnicas de hidroponia. An Acad Pernamb Ciênc Agron 2012 ;8:107-37.

31.Sánchez Laiño A, Meza Chica A, Álvarez Tubay A, Rizzo Zamora L, Guadalupe Puente A. Forraje verde hidropónico de maíz (Zea mays) deshidratado en el engorde de conejos nueva zelanda (Oryctolagus cuniculus). Ciencia y Tecnología 2010;3(2):21-3.

32.Fuentes F, Poblete C, Huerta M, Palape I. Evaluación de la producción y calidad nutritiva de avena como forraje verde hidropónico en condiciones de desierto. Idesia (Arica) 2011;29(3):7581.

33.Flores López R, Sánchez del Castillo F, Rodríguez Pérez JE, Colinas León MT, Mora Aguilar R, Lozoya Saldaña H. Densidad de población en cultivo hidropónico para la producción de tubérculo-semilla de papa (Solanum tuberosum L.). Rev Chapingo Ser Hortic 2009;15(3):251-8.

34. Vargas Rodríguez CF. Comparación productiva de forraje verde hidropónico de maíz, arroz y sorgo negro forrajero. Agromeso 2008;19(2):23340. 